\title{
Shortcomings in methodology complicate measurements of serum retinol binding protein (RBP4) in insulin-resistant human subjects
}

\author{
T. E. Graham • C. J. Wason • M. Blüher • B. B. Kahn
}

Received: 14 July 2006 / Accepted: 1 November 2006 / Published online: 9 February 2007

(C) Springer-Verlag 2007

\begin{abstract}
Aims/hypothesis Levels of retinol binding protein (RBP4) are increased in the serum of insulin-resistant human subjects even before overt diabetes develops. RBP4 levels correlate with insulin resistance, BMI, WHR, dyslipidaemia and hypertension. Improvement of insulin sensitivity with exercise training is associated with reduction in serum RBP4 levels. Therefore serum RBP4 may be useful for early diagnosis of insulin resistance and for monitoring improvements in insulin sensitivity. We sought to determine the performance of assays for this application.

Subjects and methods We compared quantitative western blotting and three commercially available multiwell immunoassays in parallel measurements of RBP4 concentrations in serum from insulin-sensitive subjects and from insulinresistant subjects with impaired glucose tolerance or type 2 diabetes.

Results The assays yielded different absolute values and magnitudes of elevation of serum RBP4. Western blotting and a sandwich ELISA reported RBP4 concentrations that highly inversely correlated with insulin sensitivity measured by euglycaemic-hyperinsulinaemic clamp. However, western blotting yielded concentrations with a greater dynamic range and less overlap between control and insulin-resistant subjects. Two competitive enzyme-linked immunoassays undervalued serum RBP4 concentrations in
\end{abstract}

T. E. Graham • C. J. Wason • B. B. Kahn $(\bowtie)$

Division of Endocrinology, Diabetes and Metabolism,

Beth Israel Deaconess Medical Center,

99 Brookline Avenue/Research North, Rm. 380C,

Boston, MA 02215, USA

e-mail: bkahn@bidmc.harvard.edu

M. Blüher

Department of Medicine, University of Leipzig Medical Center,

Leipzig, Germany insulin-resistant subjects, possibly due to assay saturation. Poor linearity of dilution also limited assay utility. All assays tested exhibited greater immunoreactivity with urinary (C-terminal proteolysed) RBP4 than with fulllength RBP4, the predominant form in serum.

Conclusions/interpretations These findings support the use of quantitative western blotting standardised to full-length RBP4 protein as a 'gold standard' method for measuring serum RBP4 in insulin-resistant states. Other assays should use full-length RBP4 and be extensively cross-validated using other methods.

Keywords Adipokine - Insulin-resistance .

Metabolic syndrome · Obesity · Type 2 diabetes $\cdot$ Vitamin A

$\begin{array}{ll}\text { Abbreviations } & \\ \text { EIAs } & \text { enzyme-linked immunoassays } \\ \text { GDR } & \begin{array}{l}\text { glucose disposal rate } \\ \text { matrix-assisted laser desorption } \\ \text { MALDI-TOF } \\ \text { ionisation-time of flight } \\ \text { retinol binding protein-4 }\end{array} \\ \text { RBP4 } & \begin{array}{l}\text { transthyretin } \\ \text { TTR }\end{array}\end{array}$

\section{Introduction}

Insulin resistance plays a key role in the development of type 2 diabetes. Even without hyperglycaemia, insulin resistance confers increased risk of cardiovascular disease and early mortality [1-3]. The term metabolic syndrome denotes a constellation of insulin resistance and cardiovascular risk factors such as hypertension, hypertriglyceridaemia, and low HDL-cholesterol [4]. While obesity is the most common cause of insulin resistance and the metabolic 
syndrome, lean individuals may be insulin-resistant, frequently due to genetic background [5].

We recently reported that serum retinol binding protein (RBP4) is increased in insulin-resistant mice [6] and in a variety of insulin-resistant human subjects with obesity, impaired glucose tolerance or type 2 diabetes, and even in lean, normoglycaemic subjects with strong family histories of type 2 diabetes [7]. Furthermore, serum RBP4 levels correlate inversely with insulin sensitivity measured by the euglycaemic-hyperinsulinaemic clamp technique. Serum RBP4 levels fell in subjects in whom exercise training improved insulin sensitivity and not in those who showed no improvement in insulin sensitivity [7]. Therefore, serum RBP4 may provide both a useful marker and a relative index of the severity of insulin resistance among individuals with a variety of clinical presentations.

Commercial assays for measuring serum or urine RBP4 were originally manufactured for assessing vitamin A (retinol) status. While upper limits for RBP4 levels in normal subjects have not been established, minimum RBP4 concentrations are reported to be $\sim 15$ to $30 \mu \mathrm{g} / \mathrm{ml}$ among healthy subjects without vitamin A deficiency $[8,9]$. In vitamin A deficiency, liver stores of retinol are depleted $[10,11]$. Since RBP4 must be bound to retinol for efficient secretion from hepatocytes [12], vitamin A deficiency reduces serum RBP4. Consequently, serum RBP4 concentrations correlate well with other indicators of vitamin A status, including serum retinol concentrations measured by HPLC [8] or total body retinol status measured by retinol stable isotope dilution methods [13, 14]. Until recently, the potential value of measuring RBP4 levels in the setting of insulin resistance had not been recognised, although elevations in serum and urinary retinol or RBP4 levels have been reported in humans with type 2 diabetes $[15,16]$.

Several commercial RBP4 assays use standard curves with ranges that could accommodate measurements of increased concentrations of serum RBP4 as observed in insulin resistance. However, these assays have not been validated for this application. Therefore, we sought to compare the performance of several methods for measuring RBP4. We analysed serum RBP4 from insulin-resistant subjects with impaired glucose tolerance or type 2 diabetes and from insulin-sensitive control subjects with normal glucose tolerance using three commercial assays and a quantitative western blotting assay previously validated for measuring serum RBP4 in insulin-resistant subjects [7]. We found considerable discrepancy among the assays, particularly for serum RBP4 measurements in insulin-resistant subjects. We identify several factors that may influence assay performance, including assay saturation due to high concentrations of RBP4, poor linearity of dilution, and preferential reactivity of antibodies with the urinary form more than the serum form of RBP4. Urinary RBP4 is frequently used as a standard in commercial assays.

\section{Subjects and methods}

Subject characteristics Subjects were newly diagnosed as having normal glucose tolerance, impaired glucose tolerance or type 2 diabetes on the basis of fasting glucose and 75-g oral glucose tolerance tests [17]. Subjects had never been treated with glucose-lowering medications or insulin. They were a subset of a larger cohort described elsewhere $[7,17]$, and were selected to provide a wide range of insulin sensitivities, as determined by glucose disposal rate (GDR) measured by the euglycaemic-hyperinsulinaemic clamp with an insulin infusion rate of $20 \mathrm{mU} \mathrm{kg}^{-1} \mathrm{~min}^{-1}$ [17]. The relationship between serum RBP4 and GDR in this group was previously found to be independent of age and BMI [7]. Age and BMI were not considerations in the selection of the subjects in the present study, since the main purpose of the study was to compare the performance of different assays in measuring serum RBP4 concentrations. The present study consisted of control subjects with normal glucose tolerance (three male, three female), subjects with impaired glucose tolerance (six male, four female), and subjects with type 2 diabetes (four male, five female) from a vitamin A-sufficient region. Mean values and ranges for age, BMI, $\mathrm{HbA}_{1 \mathrm{c}}$ and GDR are shown in the legend to Table 1. The study was approved by the Ethics Committee of the University of Leipzig and written informed consent was obtained from each subject.

Serum collection and storage Serum samples were collected after overnight fasting using standard clinical venipuncture methods and glass vacuum collection tubes without clot-activating beads, protease inhibitors, or anticoagulants. Samples were centrifuged and serum supernatant fractions collected according to standard clinical laboratory procedures. Samples were divided into aliquots in polypropylene microtubes for long-term storage at $-80^{\circ} \mathrm{C}$; two freezethaw cycles were made in the process of collection and dividing into aliquots for storage. Measurements were performed on samples that were thawed and analysed simultaneously using each assay method. Standard vacuum collection tubes containing heparin, EDTA or sodium citrate were used for plasma preparation.

Quantitative western blotting Full-length recombinant RBP4 was expressed in E. coli, purified, and retinolbinding and transthyretin (TTR)-binding were verified as described [6]. Proper disulfide bond formation was verified by matrix-assisted laser desorption ionisation-time of flight (MALDI-TOF) mass spectrometry. Protein concentration was measured by the Lowry method (DC kit; BioRad, Hercules, CA, USA) which reported concentrations comparable to the Bradford method (BioRad); however, the bicinchoninic acid/ $\mathrm{CuSO}_{4}$ method underestimated 
Table 1 Comparison of assay performance

\begin{tabular}{|c|c|c|c|c|c|c|c|c|c|c|c|c|}
\hline & \multicolumn{12}{|c|}{ Assay characteristics } \\
\hline & \multicolumn{3}{|c|}{ Quantitative western blotting } & \multicolumn{3}{|c|}{ Sandwich ELISA } & \multicolumn{3}{|c|}{ Competitive EIA no. 1} & \multicolumn{3}{|c|}{ Competitive EIA no. 2} \\
\hline & $\mathrm{NGT}^{\mathrm{a}}$ & $\mathrm{IGT}^{\mathrm{b}}$ & $\mathrm{T} 2 \mathrm{D}^{\mathrm{c}}$ & $\mathrm{NGT}^{\mathrm{a}}$ & $\mathrm{IGT}^{\mathrm{b}}$ & $\mathrm{T} 2 \mathrm{D}^{\mathrm{c}}$ & $\mathrm{NGT}^{\mathrm{a}}$ & $\mathrm{IGT}^{\mathrm{b}}$ & $\mathrm{T} 2 \mathrm{D}^{\mathrm{c}}$ & $\mathrm{NGT}^{\mathrm{a}}$ & $\mathrm{IGT}^{\mathrm{b}}$ & $\mathrm{T} 2 \mathrm{D}^{\mathrm{c}}$ \\
\hline RBP4 ( $\mu \mathrm{g} / \mathrm{ml}$, range) & $\begin{array}{l}23 \pm 8 \\
(15-35)\end{array}$ & $\begin{array}{l}64 \pm 39 \\
(30-151)\end{array}$ & $\begin{array}{l}72 \pm 33 \\
(31-132)\end{array}$ & $\begin{array}{l}34 \pm 9 \\
(25-49)\end{array}$ & $\begin{array}{l}66 \pm 26 \\
(37-114)\end{array}$ & $\begin{array}{l}65 \pm 24 \\
(31-104)\end{array}$ & $\begin{array}{l}25 \pm 4 \\
(20-30)\end{array}$ & $\begin{array}{l}37 \pm 9 \\
(23-58)\end{array}$ & $\begin{array}{l}42 \pm 13 \\
(25-67)\end{array}$ & $\begin{array}{l}34 \pm 5 \\
(25-39)\end{array}$ & $\begin{array}{l}36 \pm 6 \\
(28-43)\end{array}$ & $\begin{array}{r}28 \pm 12 \\
(6-40)\end{array}$ \\
\hline Intra-assay CV (\%) & \multirow{2}{*}{\multicolumn{3}{|c|}{$\begin{array}{l}10.4 \pm 4 \\
15.6 \pm 6\end{array}$}} & \multicolumn{3}{|c|}{$12.5 \pm 5$} & \multicolumn{3}{|c|}{$10.9 \pm 4$} & \multicolumn{3}{|c|}{$4.6 \pm 2$} \\
\hline Inter-assay CV (\%) & & & & \multicolumn{3}{|c|}{$15.2 \pm 6$} & \multicolumn{3}{|c|}{$17.3 \pm 8$} & \multicolumn{3}{|c|}{ N.D. } \\
\hline $\begin{array}{l}\text { Pearson correlation } \\
\text { with GDR }(r)\end{array}$ & \multicolumn{3}{|c|}{$\begin{array}{c}-0.80(-0.91 \text { to }-0.58)^{\mathrm{d}} \\
p<0.001\end{array}$} & \multicolumn{3}{|c|}{$\begin{array}{c}-0.70(-0.86 \text { to }-0.43)^{\mathrm{d}} \\
p<0.001\end{array}$} & \multicolumn{3}{|c|}{$\begin{array}{c}-0.58(-0.80 \text { to }-0.25)^{\mathrm{d}} \\
p=0.002\end{array}$} & \multicolumn{3}{|c|}{$\begin{array}{c}0.11(-0.2 \text { to } 0.09)^{\mathrm{d}} \\
p=0.69\end{array}$} \\
\hline $\begin{array}{l}\text { Pearson correlation } \\
\text { with western blot } \\
\text { RBP4 }(r)\end{array}$ & \multicolumn{3}{|c|}{-} & \multicolumn{3}{|c|}{$\begin{array}{c}0.80(0.62 \text { to } 0.90)^{\mathrm{d}} \\
p<0.001\end{array}$} & \multicolumn{3}{|c|}{$\begin{array}{c}0.73(0.51 \text { to } 0.87)^{\mathrm{d}} \\
p<0.001\end{array}$} & \multicolumn{3}{|c|}{$\begin{array}{c}-0.47(-0.71 \text { to }-0.25)^{\mathrm{d}} \\
p=0.009\end{array}$} \\
\hline $\begin{array}{l}\text { Assay dynamic range } \\
(\% \text { GDR dynamic } \\
\text { range })\end{array}$ & \multicolumn{3}{|c|}{79} & \multicolumn{3}{|c|}{50} & \multicolumn{3}{|c|}{38} & \multicolumn{3}{|c|}{33} \\
\hline
\end{tabular}

${ }^{a}$ Age $29 \pm 7$ years, BMI $25 \pm 2 \mathrm{~kg} / \mathrm{m}^{2}$, GDR $79 \pm 19 \mathrm{mg} /(\mathrm{kg} \times \mathrm{min}), \mathrm{HbA}_{1 \mathrm{c}} 5.3 \pm 0.1 \%$

${ }^{\mathrm{b}}$ Age $55 \pm 9$ years, BMI $29 \pm 2 \mathrm{~kg} / \mathrm{m}^{2}$, GDR $17.6 \pm 9.3 \mathrm{mg} /(\mathrm{kg} \times \min ), \mathrm{HbA}_{1 \mathrm{c}} 5.7 \pm 0.1 \%$

${ }^{c}$ Age $54 \pm 6$ years, BMI $31 \pm 3 \mathrm{~kg} / \mathrm{m}^{2}$, GDR $23.4 \pm 9.9 \mathrm{mg} /(\mathrm{kg} \times \mathrm{min}), \mathrm{HbA}_{1 \mathrm{c}} 6.2 \pm 0.3 \%$

${ }^{\mathrm{d}} 95 \% \mathrm{CI}$

GDR Glucose disposal rate, $T 2 D$ type 2 diabetes

Data are means \pm SD

concentrations of purified RBP4 (not shown). Standard solutions of $15,30,60$ or $120 \mu \mathrm{g} / \mathrm{ml} \mathrm{RBP4}$ were prepared in Tris-buffered saline containing 1\% NP-40. Standards and sera were diluted 1:100 in SDS-PAGE buffer and boiled for $5 \mathrm{~min}$. Next, $15 \mu \mathrm{l}$ of diluted standards or sera and molecular weight markers were electrophoresed on $18 \%$ Tris-glycine SDS-PAGE gels (Criterion brand; BioRad) and transferred to nitrocellulose. Western blotting was performed with non-fat milk (BioRad) as a blocking agent. Blots were incubated overnight at $4^{\circ} \mathrm{C}$ with primary antibody (A0040, DAKOCytomation, DAKO USA, Carpinteria, CA, USA) diluted 1:500 or 1:1,000 and for $1 \mathrm{~h}$ at room temperature with horseradish-peroxidase-conjugated secondary antibody (GE Healthcare Bio-Sciences, Piscataway, NJ, USA) diluted 1:2,000. Bands were detected by enzymatic chemiluminescence (Pierce Biotechnology, Rockford, IL, USA) and quantified with GeneSnap software (Synoptics/Syngene, Frederick, MD, USA). A single band for RBP4 migrated at about $21 \mathrm{kDa}$. Second-order polynomial curves were used to fit the purified RBP4 standards $\left(r^{2}>0.99\right)$ for each individual gel and calculate concentrations in serum samples. This method provides absolute measurements of RBP4 concentrations with minimal gel to gel variability $(\sim 10 \% \mathrm{CV}$ for the same sample from duplicate gels). When RBP4 concentrations exceeded the upper limits of the standard curve $(120 \mu \mathrm{g} / \mathrm{ml})$, samples were diluted 1:2 and reanalysed. To avoid irregularities from gel quality or protein transfer, RBP4 standards were placed in the same position at the centre of each gel, and the four outermost lanes were not used for samples. To monitor assay quality, serum reference samples with low and high concentrations of RBP4 (28 and $110 \mu \mathrm{g} /$ $\mathrm{ml}$, respectively) were included on every gel. Blots on which reference sample values varied by more than $15 \%$ from expected values were considered defective and not used for measurements.

Commercial multiwell immunoassay kits We tested a sandwich ELISA (ALPCO Diagnostics, Salem, NH, USA) and two competitive enzyme-linked immunoassays (EIAs): EIA no. 1 was a beta test kit provided by an anonymous manufacturer; EIA no. 2 was manufactured by Scimedx (Denville, NJ, USA). The sandwich ELISA (Lot no. 28-12004; ALPCO Diagnostics) evaluated in this study differs in reagent composition and protocol from a kit (Lot no. 0914-03; ALPCO) we previously used for measuring RBP4 in insulin-resistant subjects [7] and which is no longer available. Product information for EIA no. 2 indicated it was developed for measuring serum RBP4 as a surrogate marker of vitamin A deficiency (i.e. designed to detect below-normal serum RBP4 concentrations). Each manufacturer's protocol was followed exactly. Differences in assay components are summarised in Table 2.

Analysis of full-length and urinary RBP4 proteins To characterise full-length recombinant and purified urinary RBP4 (Sigma-Aldrich, St Louis, MO, USA), $1 \mathrm{mg} / \mathrm{ml}$ stock solutions were made in phosphate buffered saline, desalted with a Ziptip (Millipore, Billerica, MA, USA), diluted in matrix buffer, spotted on a MALDI target, and analysed with an ABI4700 TOF/TOF mass spectrometer (Applied Biosystems, Foster City, CA, USA) in linear TOF mode. 
Table 2 Assay components

\begin{tabular}{lllll}
\hline & Antibody type & Immunising antigen & Competitor protein & Standard protein \\
\hline Quantitative western blotting & Polyclonal & Urinary RBP4 & Untagged RBP4 $^{\mathrm{b}}$ (full length) \\
Sandwich ELISA & Polyclonal & Urinary RBP4 $^{\mathrm{a}}$ & N/A & Urinary RBP4 $^{\mathrm{a}}$ \\
Competitive EIA no. 1 & Polyclonal & FLAG-RBP4 $^{\mathrm{f}}$ (full length) & FLAG-RBP4 $^{\mathrm{c}}$ (full length) & FLAG-RBP4 $^{\mathrm{c}}$ (full length) \\
Competitive EIA no. 2 & Monoclonal & Urinary RBP4 $^{\mathrm{a}}$ & Urinary RBP4 $^{\mathrm{a}}$ & Urinary RBP4 $^{\mathrm{a}}$ \\
\hline
\end{tabular}

${ }^{a}$ Urinary RBP4 refers to protein purified from urine of human subjects

${ }^{\mathrm{b}}$ Untagged RBP4 refers to full-length recombinant human RBP4 which does not contain a fused epitope sequence

${ }^{\mathrm{c}}$ FLAG-RBP4 refers to a fusion protein consisting of full-length RBP4 and an epitope sequence recognised by the FLAG antibody

For comparison of western blot immunoreactivity, 20 to $160 \mu \mathrm{g} / \mathrm{ml}$ stock solutions of full-length recombinant or urinary RBP4 were measured on the same blot as described above for human serum. For comparison of ELISA immunoreactivity, a serum sample known to contain $15 \mu \mathrm{g} / \mathrm{ml}$ of serum RBP4 was supplemented ('spiked') with increasing concentrations of urinary or full-length RBP4 protein. Therefore non-RBP4 serum 'matrix' components were the same for every sample. Control samples containing $15 \mu \mathrm{g} / \mathrm{ml}$ serum not supplemented with purified RBP4 protein were analysed in parallel. RBP4 concentrations were measured using the ELISA manufacturer's protocol and urinary RBP4 standards. Recovery of 'spiked' RBP4 was calculated as the concentration measured in each sample minus the quantity of RBP4 measured in the control sample.

Statistical analysis Data are expressed as means \pm SD unless otherwise noted. Simple regression analyses with Pearson correlations were determined using Analyse-It software version 1.73 (Analyse-It, Leeds, UK). Dynamic range for each assay was calculated as the average variance of each individual RBP4 concentration from the mean concentration of all the samples in that assay. Dynamic range for the GDR values measured by euglycaemic-hyperinsulinaemic clamp was calculated using the same method. Since RBP4 and GDR are measured with different units, variance was normalised as a fraction of the mean for each parameter. Since GDR values displayed a greater dynamic range than the RBP4 concentrations we expressed RBP4 dynamic range as a percentage of the GDR dynamic range (arbitrarily defined as 100\%) for each assay.

\section{Results}

Quantitative western blotting and three commercial multiwell immunoassay kits (sandwich ELISA, EIA no. 1 and EIA no. 2) were used to measure serum RBP4 from subjects with normal glucose tolerance, impaired glucose tolerance, and type 2 diabetes (Table 1). Each assay used different combinations of antibody types and purified RBP4 proteins (Table 2). Quantitative western blotting and EIA no. 1 reported RBP4 concentrations in subjects with normal glucose tolerance of $23 \pm 8$ and $25 \pm 4 \mu \mathrm{g} / \mathrm{ml}$, respectively, values consistent with some reports for normal subjects without vitamin A deficiency $(\sim 15-30 \mu \mathrm{g} / \mathrm{ml})[8,9]$. In contrast, the sandwich ELISA and competitive EIA no. 2 reported higher RBP4 concentrations in subjects with normal glucose tolerance ( $34 \pm 9$ and $34 \pm 5$, respectively).

We have previously reported that serum RBP4 levels are elevated in subjects with impaired glucose tolerance or type 2 diabetes and exhibit very little overlap with subjects with normal glucose tolerance [7]. In this study, quantitative western blotting, the sandwich ELISA, and competitive EIA no. 1 all reported increased mean concentrations of serum RBP4 in insulin-resistant subjects (Table 1); only competitive EIA no. 2 did not. However, the majority of RBP4 concentrations of subjects with normal glucose tolerance determined by the sandwich ELISA or competitive EIA kits overlapped with values of subjects with impaired glucose tolerance or type 2 diabetes; this overlap was extensive for the EIA kits (Fig. 1). In contrast, quantitative western blotting reported only one subject with normal glucose tolerance with an RBP4 concentration within the range of subjects with impaired glucose tolerance or type 2 diabetes (Fig. 1), which was consistent with our data in a larger group of subjects [7]. Therefore, quantitative western blotting may be superior for discriminating RBP4 levels in normal subjects from elevated levels in insulin-resistant subjects. This is important for determining whether RBP4 can be used for early detection of insulin resistance before clinically apparent disease.

We recently reported that serum RBP4 concentrations are highly inversely correlated with insulin sensitivity (clamp GDR), fasting insulin and insulin level during an oral glucose tolerance test [7]. To determine whether assay techniques may influence this relationship, we performed correlation analyses between RBP4 concentrations measured by each method and GDR measurements (Table 1, Fig. 2). Western blotting exhibited both the greatest dynamic range of RBP4 concentrations and the strongest inverse correlation between RBP4 concentrations and GDR 
Fig. 1 Absolute measurements of serum RBP4 made with four different techniques. RBP4 was measured in identical serum samples using quantitative western blotting or three different commercially available multiwell immunoassays: one sandwich ELISA and two different competitive enzymelinked immunoassays (EIAs) (no. 1, no. 2). For competitive EIA no. 2, the samples were initially measured exactly according to the manufacturer's instructions (Unadjusted-per protocol). Samples were then adjusted by additional dilutions (using factors of 1:2, 1:3 or 1:4), based on prior knowledge of RBP4 concentrations measured by western blotting, and re-measured with the same assay. T2D, subjects with type 2 diabetes

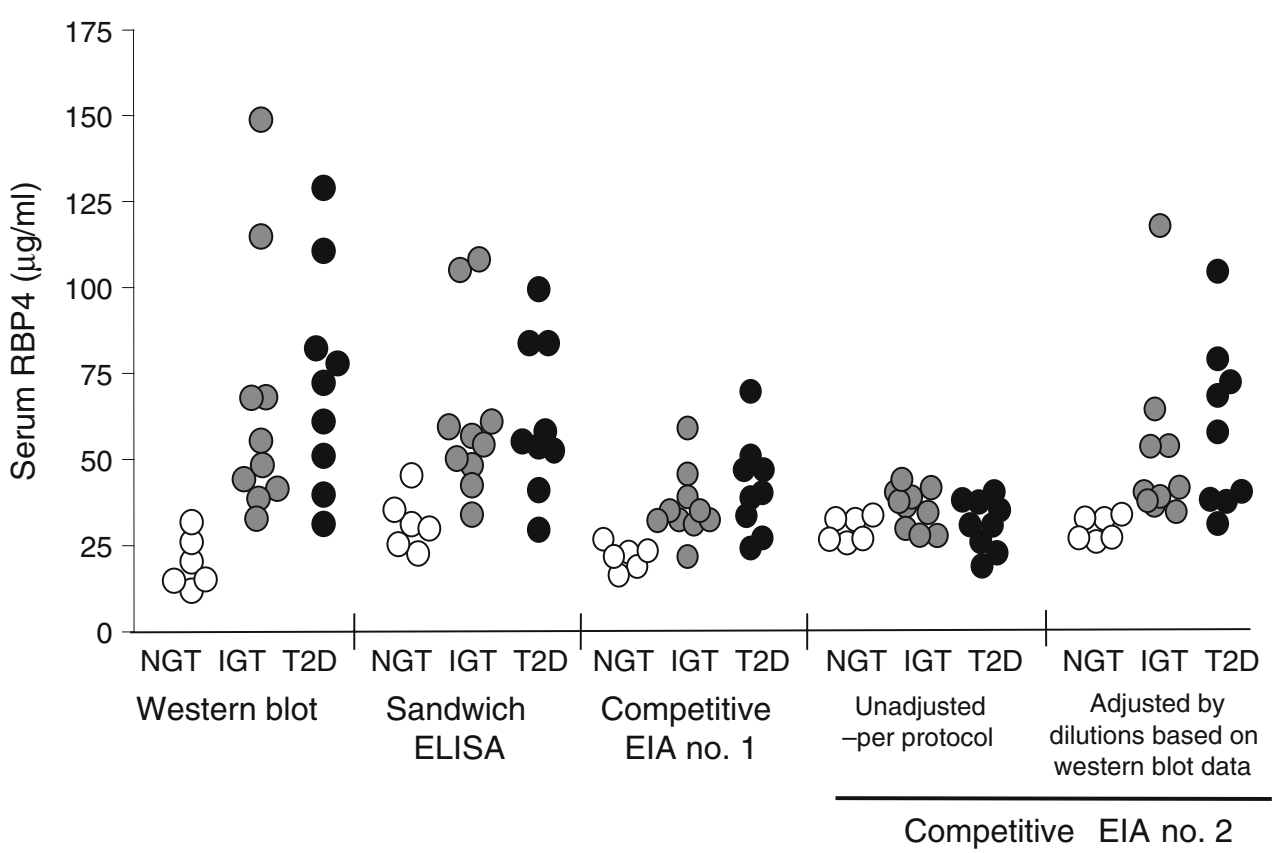

( $r=-0.80, P<0.001$; Table 1, Fig. 2a). Even though the sandwich ELISA reported higher than normal serum RBP4 concentrations in subjects with normal glucose tolerance, the concentrations overall exhibited a strong inverse correlation with GDR $(r=-0.70, p<0.001$; Table 1, Fig. 2b). However, overlap of values of subjects with normal glucose tolerance with values of subjects with impaired glucose tolerance or type 2 diabetes limits the usefulness of this assay. Competitive EIA no. 1 reported concentrations with a less strong but significant inverse correlation with insulin sensitivity $(r=-0.58, p=0.002$; Table 1). However, competitive EIA no. 2 reported RBP4 concentrations that did not correlate with GDR (Table 1). Therefore, inverse correlations between RBP4 concentrations and insulin sensitivity may be observed in some a

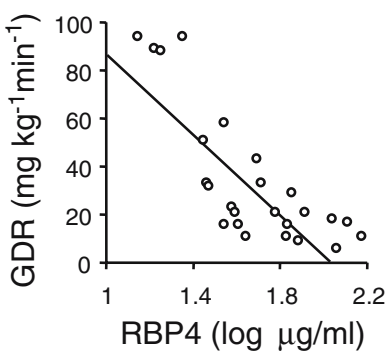

b

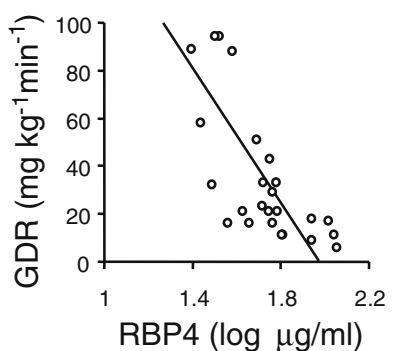

Fig. 2 Correlations between RBP4 and insulin sensitivity using quantitative western blotting (a) and ELISA (b). Serum RBP4 concentrations correlated inversely with insulin sensitivity, determined by glucose disposal rate (GDR) during a euglycaemic-hyperinsulinaemic clamp study. Analysis was performed by Pearson method. $p<0.001, r=0.80$ (a); $p<0.002, r=0.70$ (b); $p$ values indicate significance testing. 95\% CIs, see Table 1 assays even when discrepancies in absolute values of RBP4 concentrations exist.

To determine why EIA no. 2 reported consistently lower RBP4 values among insulin-resistant subjects, we examined the raw optical absorbance unit data from that assay. Absorbance values for samples fell within the standard curve and expected values reported by the manufacturer (data not shown). However, EIA no. 2 employs a standard curve with a maximum concentration $(40 \mu \mathrm{g} / \mathrm{ml})$ that is lower than RBP4 concentrations found in many insulinresistant subjects by western blotting or ELISA [7]. We hypothesised that this discrepancy might be accounted for by assay saturation and loss of competitive binding kinetics due to increased quantities of RBP4 present in sera of subjects with impaired glucose tolerance or type 2 diabetes [18]. Consistent with the notion of assay saturation, we found that RBP4 values measured by EIA no. 2 correlated negatively with RBP4 values measured by western blotting (Table 1). This observation suggests that higher actual concentrations of RBP4 produce greater saturation of the assay and lower apparent concentrations as measured by EIA no. 2.

To test for assay saturation, we lowered RBP4 concentrations in serum samples from subjects with impaired glucose tolerance or type 2 diabetes by making additional dilutions $(1: 2,1: 4$, or $1: 8)$ beyond that recommended by the manufacturer and re-measured the samples using competitive EIA no. 2. Dilution factors were selected individually for each sample based on expected RBP4 concentrations determined by quantitative western blotting. RBP4 concentrations obtained after these dilutions with EIA no. 2 (Fig. 1, adjusted) positively correlated with concentrations 
measured by western blotting ( $r=0.76$, CI $0.64-0.88, p<0.001$ ), and inversely correlated with GDR $(r=-0.70, \mathrm{CI}-0.86$ to -0.42 , $p<0.001$; data not shown), confirming that assay saturation in competitive EIA no. 2 masks elevations of serum RBP4 in insulin-resistant subjects. The intra-assay $\% \mathrm{CV}$ after adjustment $(12.1 \%)$ was greater than before adjustment $(4.6 \%)$ and similar to values for the other assays, also indicating assay saturation was overcome by the additional dilutions. We speculate that some degree of assay saturation could also contribute to lower RBP4 concentrations for subjects with impaired glucose tolerance or type 2 diabetes and the overall decreased dynamic range observed in competitive EIA no. 1 (Table 1).

We attempted to modify EIA no. 2 for measurements of elevated RBP4 in insulin-resistant subjects. We introduced an $80 \mu \mathrm{g} / \mathrm{ml}$ standard prepared from the manufacturer's calibrator protein to increase the assay range and performed serial dilutions beyond the manufacturer's recommended standard dilution (1:25). Table 3 shows analysis of samples from representative subjects with normal glucose tolerance (serum sample A) or impaired glucose tolerance (serum sample B). Linearity of dilution was absent for EIA no. 2, as the concentrations calculated for diluted samples varied as much as $75 \%$ from those obtained using the manufacturer's standard dilution (Table 3). Because raw absorbance values fell within the range of the standard curve (Table 3), there were no criteria to determine which values were correct or incorrect. Quantitative western blotting measured concentrations of 33.9 and $86.6 \mu \mathrm{g} / \mathrm{ml}$ for samples A and B, respectively (Table 3). To obtain results consistent with quantitative western blotting, widely different dilution factors would be needed for each sample (e.g. a dilution factor of 1 for sample A vs a dilution factor of 8 for sample B). Therefore, without a priori knowledge of RBP4 levels independently determined by western blotting or possibly ELISA to guide the choice of dilution factor, measurements of RBP4 in insulin-resistant subjects made with competitive assays could potentially result in erroneous conclusions. Since the relative ratios of RBP4 concentrations for samples $\mathrm{A}$ and $\mathrm{B}$ differed depending on the dilution factors used, artefact introduced by dilution could interfere with the ability to correlate serum RBP4 values with metabolic parameters such as BMI or clamp GDR.

Specific antibodies and proteins used for standard curves are likely to influence measurements of serum RBP4. Except for competitive EIA no. 1, each assay used either urinary RBP4 or antibodies raised against urinary RBP4 (Table 2). Urinary RBP4 is modified by proteolysis at the carboxyl terminus $[19,20]$ and does not bind TTR. Thus, it is filtered by the glomerulus and excreted in the urine [21]. Only a small fraction of serum RBP4 $(<10 \%)$ is proteolysed in normal subjects $[19,22,23]$. Since carboxyl-terminus amino acid differences may influence the binding of antibodies to the protein, we hypothesised that assays might exhibit different affinities for full-length serum RBP4 and proteolysed urinary RBP4.

We compared reactivity of a polyclonal antibody (DAKO A0040) raised against urinary RBP4 with recombinant full-length RBP4 produced in our laboratory [6] and

Table 3 Lack of linearity of dilution in RBP4 measurements by EIA no. 2

\begin{tabular}{|c|c|c|c|c|c|c|c|c|}
\hline \multirow[t]{2}{*}{$\begin{array}{l}\text { RBP4 standards } \\
(\mu \mathrm{g} / \mathrm{ml})\end{array}$} & \multirow[t]{2}{*}{$\begin{array}{l}\text { Dilution } \\
\text { factor }\end{array}$} & \multicolumn{2}{|c|}{ Raw absorbance (AU) } & \multicolumn{2}{|c|}{$\begin{array}{l}\text { Measured concentration } \\
(\mu \mathrm{g} / \mathrm{ml})\end{array}$} & \multirow{2}{*}{$\begin{array}{l}\text { Average } \\
\text { measured } \\
\text { concentration } \\
(\mu \mathrm{g} / \mathrm{ml})\end{array}$} & \multirow{2}{*}{$\begin{array}{l}\text { Final calculated } \\
\text { concentration based } \\
\text { on dilution } \\
(\mu \mathrm{g} / \mathrm{ml})\end{array}$} & \multirow{2}{*}{$\begin{array}{l}\text { Concentration } \\
\text { measured by } \\
\text { quantitative western } \\
\text { blotting }(\mu \mathrm{g} / \mathrm{ml})\end{array}$} \\
\hline & & 1 & 2 & 1 & 2 & & & \\
\hline 0 & 1 & 0.015 & 0.019 & & & & & \\
\hline 10 & 1 & 0.836 & 0.842 & & & & & \\
\hline 20 & 1 & 0.442 & 0.405 & & & & & \\
\hline 40 & 1 & 0.233 & 0.213 & & & & & \\
\hline 80 & 1 & 0.108 & 0.112 & & & & & \\
\hline \multirow[t]{3}{*}{ Serum sample A } & 1 & 0.271 & 0.253 & 32.6 & 34.9 & 33.8 & 33.8 & 33.9 \\
\hline & 2 & 0.359 & 0.366 & 24.6 & 24.1 & 24.4 & 48.7 & \\
\hline & 4 & 0.625 & 0.543 & 13.7 & 16.0 & 14.8 & 59.3 & \\
\hline \multirow[t]{4}{*}{ Serum sample B } & 1 & 0.146 & 0.137 & 51.2 & 54.5 & 52.9 & 52.9 & 86.6 \\
\hline & 2 & 0.276 & 0.250 & 32.1 & 35.3 & 33.7 & 67.4 & \\
\hline & 4 & 0.455 & 0.472 & 19.2 & 18.5 & 18.9 & 75.5 & \\
\hline & 8 & 0.735 & 0.767 & 10.6 & 10.2 & 10.4 & 83.2 & \\
\hline
\end{tabular}

Sample A was obtained from a control subject with normal glucose tolerance and Sample B from an insulin-resistant subject with impaired glucose tolerance. Each sample was diluted at the manufacturer's recommended dilution of 1:25 (dilution factor 1) or additionally diluted two-, four- or eightfold (dilution factor 2, 4 or 8) using the sample dilution buffer supplied with the assay. Absorbance measurements were made in duplicate for each sample with a standard spectrophotometric plate reader (raw absorbance 1 and 2) and the corresponding concentrations were determined based on the standard curve (measured concentration 1 and 2). The final calculated concentrations (last column) were obtained by multiplying the average measured concentration of the duplicates by the dilution factor. 
a

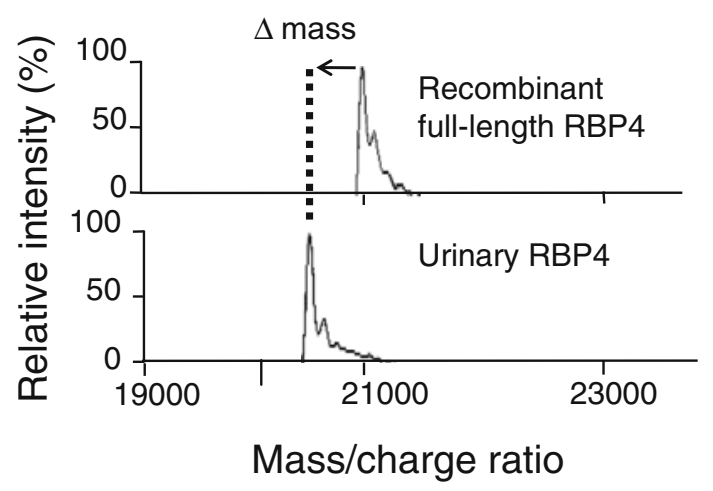

b
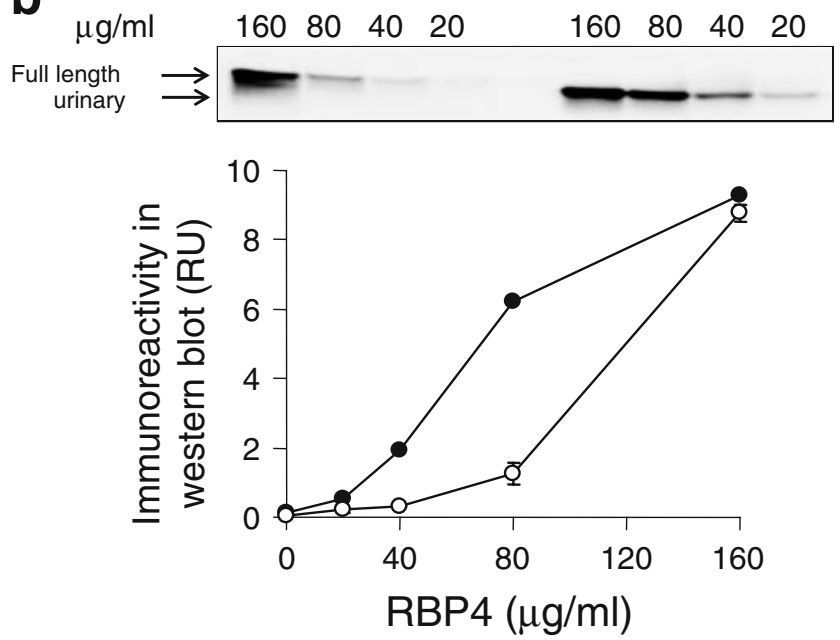

C

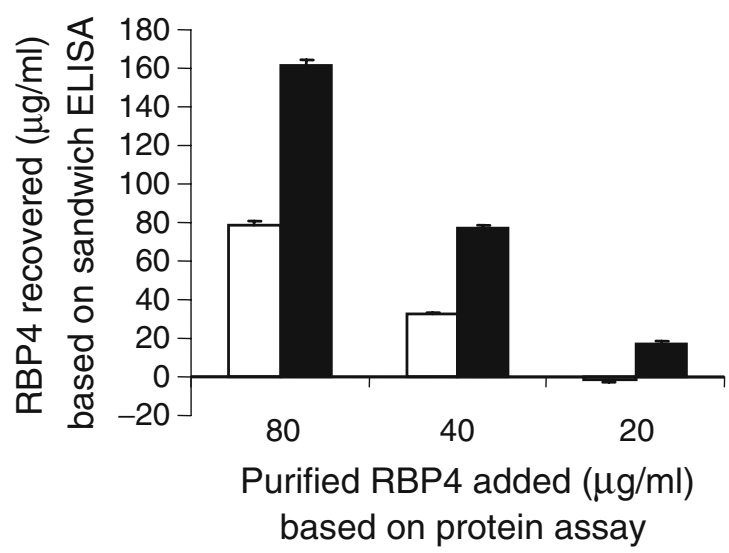

commercially available RBP4 purified from urine (Sigma). As shown in Fig. 3a, MALDI-TOF mass spectrometry confirmed that urinary RBP4 is predominately modified by proteolysis of the carboxyl terminus. The measured molecular mass of recombinant full-length RBP4, which contains an N-terminus methionine residue, was 21,197 Da, whereas the molecular mass of urinary RBP4 was 20,549 Da. Urinary RBP4 lacks an N-terminus methionine residue and the carboxyl terminus four amino acids,
Fig. 3 Differences in immunoreactivity or assay recovery between full-length recombinant and urinary RBP4 proteins. a Matrix-assisted laser desorption ionisation-time of flight (MALDI-TOF) mass spectrometry. Molecular mass peaks (mass $/$ charge $^{+1}$ ) corresponding to purified preparations of full-length serum RBP4 and urinary RBP4 were determined using MALDI-TOF mass spectrometry as described in Subjects and methods. The difference in molecular weights $(\Delta$ mass $=-648 \mathrm{Da}$ ) is accounted for by addition of an N-terminus methionine residue in full-length recombinant RBP4 and loss of the peptide $(-R N L L)$ due to proteolysis of the carboxyl terminus of urinary RBP4. b Western blotting of full-length recombinant and urinary RBP4 proteins. Equal quantities of recombinant full-length RBP4 and purified urinary RBP4 were analysed by western blotting with a polyclonal antibody raised against urinary RBP4, as described in Subjects and methods. Immunoreactive bands were imaged and luminescence was quantified and expressed as relative units $(R U)$. Filled circles, urinary (proteolysed) RBP4; open circles, recombinant full-length RBP4. c Recovery of full-length and urinary RBP4 by sandwich ELISA assay. A serum sample from a normal subject known to contain $15 \mu \mathrm{g} / \mathrm{ml}$ of serum RBP4 was supplemented ('spiked') with increasing quantities of each type of purified RBP4 protein and measured by the sandwich ELISA assay. Apparent recoveries of the purified proteins were calculated by the difference between the concentration of RBP4 measured in the RBP4-'spiked' samples and identical samples with a concentration of $15 \mu \mathrm{g} / \mathrm{ml} \mathrm{RBP4}$ that had not been 'spiked' with purified RBP4. Filled bars, urinary (proteolysed) RBP4; open bars, recombinant full-length RBP4. Values in panels $\mathbf{b}$ and $\mathbf{c}$ are means \pm SEM

-RNLL, resulting in a measured mass difference of $648 \mathrm{Da}$. Each peak contains a small shoulder peak caused by matrix particle adducts. The polyclonal antibody raised against urinary RBP4 exhibited greater western blotting reactivity for urinary RBP4 than for full-length RBP4 at several concentrations (Fig. 3b). Furthermore, the relationships of antibody reactivity to protein concentrations produced different curves for the two proteins (Fig. 3b).

We determined how the different purified proteins behave in the sandwich ELISA assay, which utilises urinary RBP4 as the standard and an antibody raised against urinary RBP4. Identical serum samples were supplemented ('spiked') with different quantities of full-length or urinary RBP4 and the ELISA kit was used to measure RBP4 (Fig. 3c). Recoveries of the added proteins were calculated as described in Subjects and methods. As shown in Fig. 3c, the ELISA kit exhibited greater reactivity for urinary RBP4 than for full-length RBP4 at all concentrations. Whereas the assay failed to detect or underestimated concentrations of full-length RBP4 at lower concentrations, it exaggerated concentrations of urinary RBP4 at higher concentrations. These data indicate that differences in the reactivity of antibodies for urinary and full-length RBP4 may influence absolute values of RBP4 measured by immunoassays. Furthermore, these data predict that levels of serum RBP4 will be underestimated by assays that use urinary RBP4 instead of full-length RBP4 as a standard. 


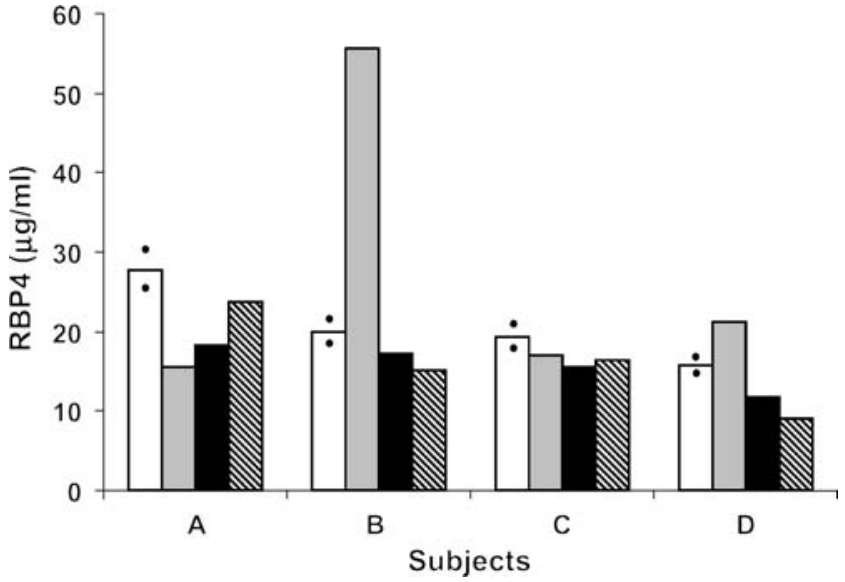

Fig. 4 Influence of sample collection methods on RBP4 measurement. Blood was collected from four normal subjects (A, B, C, D) after an overnight fast. Subjects were non-obese and had normal glucose tolerance. Following a single venipuncture, blood was collected sequentially into four standard vacuum collection tubes. Serum tubes contained no additives (open bar), and plasma tubes contained heparin (grey bar), sodium citrate (black bar) or EDTA (hatched bar). Serum samples were run in duplicate (closed circles) to establish the error of quantitative western blotting. Comparison measurements were made in single plasma samples prepared simultaneously from the same blood. The bars denote average values of the duplicate serum samples or single plasma values

In addition to assay design, we found that collection methods may influence measurements of RBP4 in blood. Plasma anticoagulants altered the absolute amount of RBP4 immunoreactivity (measured by western blotting) detected in identical blood samples of normal subjects (Fig. 4). The influence of each anticoagulant was unpredictable from subject to subject, and therefore no general rules could be established to predict plasma anticoagulant interference (Fig. 4). The effects of clot activator/serum separators should also be tested.

\section{Discussion}

Serum RBP4 is elevated in insulin resistance and may indicate risk of type 2 diabetes and cardiovascular disease $[6,7]$. Moreover, studies in mice indicate that elevated serum RBP4 can cause insulin resistance [6]. In humans, serum RBP4 concentrations correlate highly with the severity of insulin resistance, and in subjects in whom exercise training improves insulin sensitivity, serum RBP4 concentrations are lowered [7]. Therefore, accurate serum RBP4 measurements could provide a quantitative index of disease severity and a benchmark by which to follow interventions aimed at improving insulin sensitivity. Furthermore, since RBP4 levels are elevated in serum of lean, non-diabetic but insulin-resistant people with a strong genetic risk of type 2 diabetes [7], RBP4 may be useful as an early marker of diabetes risk.

In prior studies, western blotting and an ELISA sandwich assay produced RBP4 measurements that distinguished normal individuals from insulin-resistant individuals and correlated with magnitude of insulin resistance [7]. That ELISA assay is no longer available. In the present study, we found that some commercial assays report higher RBP4 levels among insulin-sensitive subjects than other methods, and the levels are higher than expected for a normal population $[8,9]$. The cause of this is not clear, but could reflect the background signal of the assay or differences in reactivity between the full-length RBP4 protein, which is the dominant form in serum, and the proteolysed urinary RBP4 used in these assays (discussed below). Therefore it seems advisable to routinely compare RBP4 levels of insulinsensitive control subjects with normative population data. We find this practice also identifies samples in which RBP4 is degraded resulting in serum levels considerably lower than the reported range of normal for a given population.

We found that competitive EIA assays may selectively underestimate serum RBP4 levels in the setting of insulin resistance due to assay saturation. Additional dilution of serum samples rectified this problem in the case of one assay (competitive EIA no. 2). However, this may not be a practical strategy for correcting these assays, since universally increasing the dilution of all the samples could lower serum RBP4 concentrations below assay range for subjects with normal glucose tolerance. Furthermore, since EIA no. 2 did not exhibit linearity of dilution (Table 3), a prior knowledge of RBP4 concentrations measured by western blotting was needed to guide the selection of the dilution factors that enabled accurate measurements (Fig. 1, adjusted values). Therefore, adaptation of competitive assays for the measurement of RBP4 in insulin-resistant subjects will require protocol modifications more extensive than increased sample dilutions and may necessitate adjustments of antibody and/or competitor protein concentrations.

An unexpected finding was that RBP4 immunoassays (quantitative western blotting and sandwich ELISA) exhibited different reactivities for full-length RBP4 and urinary RBP4 modified by carboxyl terminus-proteolysis. Since urinary RBP4 is used as a protein standard and/or competitive binding reagent in some commercial assays, these differences could influence serum RBP4 measurements. Since full-length RBP4 is the dominant form in serum [19, 22, 23], use of full-length RBP4 as a protein standard or use of monoclonal antibodies that react equally with full-length and carboxyl terminusproteolysed RBP4 would be preferable.

These findings further suggest that the abundance of proteolysed RBP4 endogenously present in serum could influence measurement of RBP4 by immunoassays. It has been proposed that RBP4 carboxyl terminus proteolysis 
occurs in circulation but results in rapid clearance of RBP4 by the kidney [23]. It is not known whether proteolysed forms of RBP4 are increased in serum in insulin resistance or type 2 diabetes without renal failure. In 60 subjects with normal glucose tolerance, impaired glucose tolerance, or type 2 diabetes without renal impairment, we found no correlation between serum RBP4 concentrations and creatinine or urinary albumin concentrations ([7]; Graham, Blüher and Kahn, unpublished data). However, since proteolysed RBP4 can accumulate in renal failure [19, 22, 23], we advise caution in interpreting serum RBP4 measurements in patients with advanced nephropathy.

Another potential cause for assay inaccuracy is interference from serum TTR. TTR circulates in a three- to fivefold molar excess over RBP4. Circulating RBP4 is highly bound to TTR in a 1:1 stoichiometric ratio, and there is little or no 'free RBP4' in circulation [24]. Using gel filtration chromatography to analyse the RBP4-TTR complex, we found that increased serum RBP4 remains bound to TTR in insulin-resistant states (not shown). Nevertheless, because the affinity of RBP4-TTR binding is very strong [24], the relative stoichiometry and affinity of the two proteins in serum could conceivably influence kinetics of RBP4antibody binding under the non-denaturing conditions employed by the commercial EIAs and ELISAs. In contrast, western blotting, which involves a denaturing SDS-PAGE step that separates RBP4 and TTR, is not affected by the RBP4-TTR interaction. The potential for TTR to interfere with RBP4 measurements by immunoassays deserves study.

Collection methods can alter RBP4 concentrations independently of measurement techniques (Fig. 4). In particular, plasma anticoagulants (e.g. heparin, EDTA, citrate or lithium chloride) may cause spurious results. Similar observations have been made by other investigators with regard to clot activating beads. Therefore, we advise use of glass vacuum tubes with no additives until optimal methods for collecting blood for RBP4 measurements are better defined. Subject characteristics that could also influence serum RBP4 include fasting/fed state and physical activity. Assays for rodents also require rigorous validation.

In conclusion, quantitative western blotting is the most reliable method for assaying serum RBP4 elevations associated with insulin resistance, because it has the greatest dynamic range and inverse correlation with insulin sensitivity (clamp GDR). Furthermore, quantitative western blotting reports RBP4 concentrations with the least overlap between insulin-sensitive and insulin-resistant subjects. A commercially available sandwich ELISA reports RBP4 concentrations that inversely correlate with insulin resistance, but values in normal subjects are higher than expected. Therefore, this assay underestimates the differences between insulin-sensitive and insulin-resistant people. Other assay methods, especially nephelometry, deserve testing. At present, we advocate the use of quantitative western blotting employing full-length RBP4 protein standards as the 'gold standard' method for measuring serum RBP4 in insulin resistance and for validating the accuracy of other assays.

Note added in proof While this paper was being prepared for publication, we obtained RBP4 measurements by nephelometry on serum from 86 subjects: 27 with NGT and 59 with IGT or type 2 diabetes. The assays were kindly run by C. Perkins at the Dade Behring Technical Support Laboratory in Newark, DE, USA (arranged by M. L. Gantzer). The correlation between RBP4 values by nephelometry and by quantitative western blotting was $r=0.63$ $(p<0.0001)$. The correlation between glucose disposal rate by euglycaemic clamp (measured in 74 of the 86 subjects) and RBP4 levels by nephelometry was $r=0.49(p<0.0001)$ compared with $r=0.80(p<0.0001)$ for quantitative western blotting.

Acknowledgements The authors wish to acknowledge A. Oberbach for euglycaemic-hyperinsulinaemic clamp measurements. This study was supported by grants from the National Institutes of Health: R01 DK-43051 (to B. B. Kahn), K08 DK-69624 (to T. E. Graham); and from the Deutsche Forschungsgemeinschaft (German Research Foundation) BL 580/3-1 (to M. Blüher) and the Takeda Pharmaceutical Company (to B. B. Kahn).

Duality of interest T. E. Graham and B. B. Kahn are listed as inventors on patent applications related to RBP4, and the latter also receives grant support from Takeda Pharmaceutical Company.

\section{References}

1. Ford ES (2005) Risks for all-cause mortality, cardiovascular disease, and diabetes associated with the metabolic syndrome: a summary of the evidence. Diabetes Care 28:1769-1778

2. Reaven GM (2005) Insulin-resistance, the insulin-resistance syndrome, and cardiovascular disease. Panminerva Med 47:201-210

3. Cersosimo E, Defronzo RA (2006) Insulin-resistance and endothelial dysfunction: the road map to cardiovascular diseases. Diabetes Metab Res Rev 22:423-426

4. Grundy SM, Brewer HB Jr, Cleeman JI, Smith SC Jr, Lenfant C (2004) Definition of metabolic syndrome: report of the National Heart, Lung, and Blood Institute/American Heart Association conference on scientific issues related to definition. Arterioscler Thromb Vasc Biol 24:e13-e18

5. Vaag A, Lehtovirta M, Thye-Ronn P, Groop L (2001) Metabolic impact of a family history of type 2 diabetes. Results from a European multicentre study (EGIR). Diabet Med 18:533-540

6. Yang Q, Graham TE, Mody N et al (2005) Serum retinol binding protein contributes to insulin-resistance in obesity and type 2 diabetes. Nature 436:356-362

7. Graham TE, Yang Q, Bluher M et al (2006) Retinol-binding protein 4 and insulin-resistance in lean, obese, and diabetic subjects. N Engl J Med 354:2552-2563

8. Gamble MV, Ramakrishnan R, Palafox NA, Briand K, Berglund L, Blaner WS (2001) Retinol binding protein as a surrogate measure for serum retinol: studies in vitamin A-deficient children from the Republic of the Marshall Islands. Am J Clin Nutr 73:594-601 
9. de Pee S, Dary O (2002) Biochemical indicators of vitamin A deficiency: serum retinol and serum retinol binding protein. J Nutr 132:2895S-2901S

10. Flores H, de Araujo CR (1984) Liver levels of retinol in unselected necropsy specimens: a prevalence survey of vitamin A deficiency in Recife, Brazil. Am J Clin Nutr 40:146-152

11. Olson JA, Gunning DB, Tilton RA (1984) Liver concentrations of vitamin $\mathrm{A}$ and carotenoids, as a function of age and other parameters, of American children who died of various causes. Am J Clin Nutr 39:903-910

12. Bellovino D, Apreda M, Gragnoli S, Massimi M, Gaetani S (2003) Vitamin A transport: in vitro models for the study of RBP secretion. Mol Aspects Med 24:411-420

13. Haskell MJ, Handelman GJ, Peerson JM et al (1997) Assessment of vitamin A status by the deuterated-retinol-dilution technique and comparison with hepatic vitamin A concentration in Bangladeshi surgical patients. Am J Clin Nutr 66:67-74

14. Tanumihardjo SA (2004) Assessing vitamin A status: past, present and future. J Nutr 134:290S-293S

15. Basualdo CG, Wein EE, Basu TK (1997) Vitamin A (retinol) status of first nation adults with non-insulin-dependent diabetes mellitus. J Am Coll Nutr 16:39-45

16. Abahusain MA, Wright J, Dickerson JW, de Vol EB (1999) Retinol, alpha-tocopherol and carotenoids in diabetes. Eur J Clin Nutr 53:630-635

17. Oberbach A, Tonjes A, Kloting $\mathrm{N}$ et al (2006) Effect of a 4 week physical training program on plasma concentrations of inflammatory markers in patients with abnormal glucose tolerance. Eur J Endocrinol 154:577-585

18. Zettner A (1973) Principles of competitive binding assays (saturation analysis). 1. Equilibrium techniques. Clin Chem 19:699-705

19. Kiernan UA, Tubbs KA, Nedelkov D, Niederkofler EE, Nelson RW (2002) Comparative phenotypic analyses of human plasma and urinary retinol binding protein using mass spectrometric immunoassay. Biochem Biophys Res Commun 297:401-405

20. Kiernan UA, Tubbs KA, Nedelkov D, Niederkofler EE, McConnell E, Nelson RW (2003) Comparative urine protein phenotyping using mass spectrometric immunoassay. J Proteome Res 2:191-197

21. Naylor HM, Newcomer ME (1999) The structure of human retinol-binding protein (RBP) with its carrier protein transthyretin reveals an interaction with the carboxy terminus of RBP. Biochemistry 38:2647-2653

22. Jaconi S, Rose K, Hughes GJ, Saurat JH, Siegenthaler G (1995) Characterisation of two post-translationally processed forms of human serum retinol-binding protein: altered ratios in chronic renal failure. J Lipid Res 36:1247-1253

23. Jaconi S, Saurat JH, Siegenthaler G (1996) Analysis of normal and truncated holo- and apo-retinol-binding protein (RBP) in human serum: altered ratios in chronic renal failure. Eur $\mathrm{J}$ Endocrinol 134:576-582

24. Zanotti G, Berni R (2004) Plasma retinol-binding protein: structure and interactions with retinol, retinoids, and transthyretin. Vitam Horm 69:271-295 